\title{
Original
}

\section{Influencia de los requerimientos ergonómicos y recursos preventivos percibidos en el desarrollo de bajas laborales por patología no traumática del hombro}

\section{Influence of ergonomic requirements and perceived preventive work resources on sickness absence due to non-traumatic shoulder disorders}

Gloria M. Rodríguez-Blanes', José Rafael Lobato-Cañón ${ }^{2,3}$, José Sánchez-Payád, José Ramón Ausó-Pérez ${ }^{5}$, Antonio Francisco J. Cardona-Llorens ${ }^{2}$

\author{
1. Centro de Salud Pública de Alcoy, Alicante. Consellería de Sanidad Universal y Salud Pública, Generalitat \\ Valenciana. España. \\ 2. Departamento de Patología y Cirugía. Universidad Miguel Hernández, San Juan de Alicante, Alicante. España. \\ 3. Instituto Nacional de la Seguridad Social, Alicante, España. \\ 4. Servicio de Medicina Preventiva. Hospital General Universitario de Alicante; Instituto de Investigación Sanitaria y \\ Biomédica de Alicante (ISABIAL), Alicante, España. \\ 5. Servicio de Cirugía Ortopédica y Traumatología, Hospital de Villajoyosa, Alicante. España.
}

\section{Recibido: 08-03-2019}

Aceptado: 04-06-2019

\section{Agradecimientos}

A todas las personas que accedieron a participar en el estudio.

\section{Correspondencia}

G.M. Rodríguez-Blanes

Centro de Salud Pública de Alcoy

Avda. Alameda de Camilo Sesto, n ${ }^{\circ} 41$

03803 Alcoy, Alicante. España

Correo electrónico: g.rodriguez@umh.es

Resumen

Introducción: El hombro doloroso es un problema médico común en Atención Primaria de salud y en el ámbito laboral. Puede producir una discapacidad funcional considerable y requerir de periodos de incapacidad temporal.

Objetivos: Describir las características de pacientes atendidos por patología no traumática del hombro, así como estudiar la asociación entre requerimientos ergonómicos y conocimientos preventivos del puesto de trabajo y el desarrollo de bajas laborales.

Material y Método: Estudio observacional transversal en una población de 345 pacientes en edad laboral que consultaron al médico de familia por dolor en el hombro en un año, pertenecientes a un centro de salud del Departamento de Salud de Alicante-Hospital General. Se utilizó un cuestionario con preguntas sobre conocimientos en prevención de riesgos laborales, requerimientos del puesto de trabajo y haber requerido o no periodos de incapacidad temporal derivada. Se realizó un análisis descriptivo y analítico.

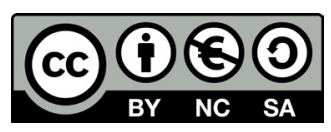

https://creativecommons.org/licenses/by-nc-sa/4.0/ 
Resultados: El 39,1\% de los entrevistados refirieron periodos de baja laboral, calificándose el $87 \%$ como enfermedad común. El 51\% refirió no tener información sobre los riesgos asociados a sus puestos de trabajo. Los requerimientos ergonómicos más frecuentes referidos por los encuestados fueron: posturas forzadas, alzar el brazo por encima del hombro, girar cuello-columna, y movimientos repetidos. Presentaron mayor probabilidad de baja las actividades relacionadas con construcción, agricultura, ganadería y pesca, conocer la existencia de evaluación de riesgos y/o plan de prevención y los requerimientos de alzar los brazos, golpear superficies, manejar cargas pesadas, y usar fuerza física. Conocer la existencia de un plan de prevención se mostró como factor de riesgo independiente (OR 4,2 [1,1-15,9]).

Conclusiones: Es importante abordar preventivamente los requerimientos ergonómicos del puesto así como conseguir mayores conocimientos de las estructuras preventivas para evitar el desarrollo de bajas laborales por hombro doloroso.

Med Segur Trab (Internet). 2019;65(255):101-11

Palabras clave: Bajas laborales, trastornos musculoesqueléticos, dolor de hombro, prevención, riesgos laborales.

Abstract

Introduction: Painful shoulder is a common medical problem in Primary Health Care and at the workplace. It can cause a considerable functional disability and can require periods of sick leave.

Objectives: To describe the characteristics of patients treated for non-traumatic diseases of the shoulder, as well as to know the association between ergonomic requirements and preventive knowledge about the workplace in the development of sickness absence.

Methods: Cross-sectional observational study in working age population of 345 patients who consulted their general practitioner throughout the year for shoulder pain, belonging to a health center from the Health Department of the Hospital General de Alicante. A questionnaire was used with questions about knowledge in occupational risk prevention, work requirements and whether or not temporary disability periods were required. A descriptive and analytical analysis was carried out.

Results: $39.1 \%$ of the interviewees reported periods of sick leave, qualified in $87 \%$ as a common disorder. $51 \%$ of them reported having no information about risks associated with their jobs. The most frequent ergonomic requirements referred by the respondents were: unnatural body posture, to raise the arm over the shoulder, to turn the neck-column, and to repeat movements. The probability of temporal disability was higher in those activities related to construction, agriculture, livestock and fisheries, people with knowledge of the existence of risk assessment and / or prevention plan, and the requirements of raising the arms, hitting surfaces, handling heavy loads, and using physical strength. To have knowledge about the existence of a prevention plan was shown as an independent risk factor (OR 4.2 [1.1-15.9]).

Conclusions: It is important to preventively address the ergonomic requirements of the position as well as to gain greater knowledge of preventive structures in order to prevent the development of sick leave due to shoulder pain.

Med Segur Trab (Internet). 2019;65(255):101-11

Keywords: sickness absence, musculoskeletal disorders, shoulder pain, prevention, occupational health. 


\section{INTRODUCCIÓN}

De manera global, los trastornos musculoesqueléticos representan el problema de salud relacionado con el trabajo más prevalente en Europa, afectando al 53\% de los trabajadores $^{1}$, y suponen la principal causa de enfermedad profesional ${ }^{2}$. En España, un $77,5 \%$ de los trabajadores se quejan de dolores o molestias musculoesqueléticos achacados a posturas y esfuerzos derivados del trabajo que realizan, y un $13,8 \%$ se refieren específicamente al hombro ${ }^{3}$. Muchas de estas dolencias pueden derivar en patologías de mayor gravedad, incapacitando al trabajador para muchos tipos de trabajo. Según la Comisión Europea, los trastornos musculoesqueléticos son la causa de la mayoría de las ausencias laborales (49,9\% de todas las ausencias de más de 3 días) y de los casos de Incapacidad Permanente para trabajar $(60 \%)^{1}$. En España fueron la principal causa de Incapacidad Temporal (IT) en 2007, produciendo más de 39 millones de días de baja laboral (23\% del coste por IT) ${ }^{4}$. Según estimaciones económicas, suponen el $40 \%$ de los costes sanitarios relacionados con el trabajo en el mundo 5 , y entre un $0,5 \%$ y un $2 \%$ del Producto Interior Bruto europeo ${ }^{2}$. Refiriéndonos específicamente a la patología del hombro, una buena estimación de los costes del tratamiento es compleja por la falta de información sobre los detalles de los tratamientos realizados, aunque se estima que los costes indirectos por bajas laborales pueden suponer entre el $50-84 \%$ del total de costes ${ }^{6,7,8}$.

El dolor de hombro es un problema médico común en la consulta de Atención Primaria (AP), siendo el tercer motivo de consulta más frecuente9. Además, en muchos casos, el médico de familia es el primer profesional sanitario consultado ${ }^{10}$. Tiene una prevalencia aproximada de un 7-26 \% de la población general en edad adulta y una incidencia entre 9-25 casos por 1000 habitantes/año9,11. Dentro del concepto más amplio de hombro doloroso podemos encuadrar la patología no traumática de la articulación del hombro, cuya principal causa son las lesiones del manguito rotador, responsables del 65-70\% de los $\operatorname{casos}^{12,13}$. Además, el dolor de hombro puede ocasionar un importante impacto en la calidad de vida percibida $^{14}$, y producir una discapacidad funcional considerable, debido a su notable influencia sobre las ausencias laborales por enfermedad, el uso de asistencia sanitaria y la retirada prematura del mercado laboral ${ }^{15}$.

El hombro doloroso tiene una etiología multifactorial, siendo también frecuente en el ámbito laboral, estimándose una fracción atribuible poblacional por exposiciones laborales en torno al $24 \%{ }^{13}$. La Organización Mundial de la Salud considera que los trastornos musculoesqueléticos del hombro en ocasiones pueden asociarse fundamentalmente al trabajo, considerándose entonces "enfermedades relacionadas con el trabajo"16, y también más de un tercio de los pacientes que acuden al centro de salud consideran su patología relacionada o agravada por su trabajo ${ }^{10}$.

El diagnóstico precoz y un tratamiento adecuado disminuyen el dolor, mejoran la funcionalidad y reducen el riesgo de incapacidad a largo plazo'. Además, los médicos de AP tienen un papel clave en la detección y manejo de las enfermedades relacionadas con el trabajo ${ }^{10}$.

Por todo ello, es de gran importancia conocer los factores que podrían intervenir en la patología no traumática del hombro para su prevención, y evitar así la necesidad de requerir periodos de baja laboral, y posibles efectos a largo plazo que puedan condicionar la actividad laboral futura. Los objetivos de este estudio fueron describir las características de los pacientes atendidos desde AP con patología no traumática del hombro; y estudiar la asociación entre los requerimientos ergonómicos y conocimientos preventivos del puesto de trabajo y el desarrollo de bajas laborales derivadas.

\section{MATERIAL Y MÉTODOS}

Diseño y Población de Estudio: Se realizó un estudio observacional transversal en población en edad laboral atendida durante un año en las consultas de un centro de atención primaria de salud del Departamento de Salud de Alicante-Hospital General. 
Criterios de Inclusión: Hombres y mujeres entre 16 y 64 años, que hayan consultado a su médico de familia por dolor a nivel del hombro a lo largo del año en el centro de salud. Las enfermedades incluidas en el estudio fueron: enfermedades por fatiga e inflamación de las vainas tendinosas, de tejidos peritendinosos e inserciones musculares y tendinosas en el hombro (tendinitis, rotura de tendones, síndrome subacromial), enfermedades de las bolsas serosas debida a la presión (bursitis del hombro) y dolor articular del hombro. Los códigos CIE-9-MC incluidos: 726.1, 726.10, 726.11, 726.12, 726.13, 726.19, 726.2, 719.01, 719.41, 719.61, 719.81, 719.91, 840.3-840.6. Se excluyeron: personas de edades $<16$ años o $\geq 65$ años; diagnósticos tras traumatismo directo y de origen artrósico en el hombro; y no haber desempeñado actividad laboral remunerada durante al menos un año anterior.

Variables: las variables que se analizaron en el estudio son (Tabla I):

Datos personales: incluyendo Sexo, Edad y Nivel de estudios.

Puesto de Trabajo: Puesto actual que se desempeña, clasificado por Ocupación y Actividad económica. Horas de trabajo diarias referidas, y Horas semanales referidas.

Conocimientos sobre prevención de riesgos laborales en las empresas: Información sobre los riesgos laborales del puesto, empleo de Equipos de Protección Individual (EPI), Servicio de Prevención de Riesgos Laborales (SPRL) asociado a la empresa, Evaluación de riesgos, Plan de prevención, Vigilancia de la salud periódica.

Requerimientos ergonómicos del puesto (basado en descripciones de tareas obtenidas de los propios pacientes en relación a su trabajo): empleo de Posturas forzadas, utilización de Brazos y Elevación de los mismos, Herramientas vibratorias, Posturas de trabajo, Manejo de cargas, Golpear superficies y empleo de Fuerza, Movimientos repetidos, trabajo Monótono y la existencia de Pausas y su duración.

Patología: existencia o no de Bajas laborales y tipo de Contingencia de la patología.

Procedimiento de recogida de datos: Se obtuvieron un total de 514 posibles casos que cumplían los criterios de inclusión, de los que 345 conformaron nuestra población final de estudio. La recogida de datos se realizó entre febrero de 2008 y mayo de 2009. A los participantes se les suministró una encuesta semiestructurada, tras informar del motivo de la recogida de datos y obtener su consentimiento informado. Las exclusiones correspondieron a 54 posibles casos que no cumplían el criterio de actividad laboral, 56 personas con las que no fue posible contactar por no disponer de teléfono de contacto o ser erróneo, 53 con los que no se pudo contactar tras 3 intentos de llamada repetidos en 3 días diferentes, 5 posibles casos se excluyeron tras manifestar no querer participar, y 1 caso por defunción por otra causa no relacionada (Figura 1 ).

Análisis de datos: Se realizó un análisis descriptivo basado en frecuencias y porcentajes. Para los análisis posteriores, los casos se dividieron en dos grupos, en función de si la persona había requerido o no estar de baja laboral durante el proceso de su patología. La asociación entre haber presentado baja laboral y las variables sociodemográficas, de prevención de riesgos laborales, y de requerimientos ergonómicos se estudió mediante la prueba de la Ji cuadrado $\left(\chi^{2}\right)$. Para estimar la magnitud de la asociación se calculó la Odds Ratio (OR) con un intervalo de confianza al 95\%. Para conocer el efecto independiente de las variables explicativas que resultaron estadísticamente significativas en el análisis bivariante, se realizó un análisis multivariante tipo regresión logística. El nivel de significación estadística utilizado en los contrastes de hipótesis fue de $p<0,05$. El cálculo de los tests estadísticos se realizó con el programa SPSS ${ }^{\circledR}$ v.15 de SPSS INC.

Consideraciones éticas: A todos los posibles casos se les informó del motivo del estudio y se solicitó su consentimiento informado a participar, no admitiéndose la posibilidad de consentimiento por representación. Asimismo, se les informó de la posibilidad revocar su consentimiento en cualquier momento de la entrevista. Para 
garantizar la confidencialidad y el anonimato de los participantes se eliminaron los datos identificativos personales, asignando a cada caso un número consecutivo de orden.

Tabla I. Principales características de los casos analizados ( $\mathrm{n}=345$ )

\begin{tabular}{|c|c|c|c|}
\hline & n (\%) & & n (\%) \\
\hline SEXO & & VIGILANCIA DE SALUD & \\
\hline Hombre.. & $111(32,2 \%)$ & Sí. & $184(53,3 \%)$ \\
\hline 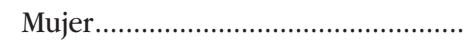 & $234(67,8 \%)$ & POSTURAS FORZADAS & \\
\hline EDAD & & Sí.... & $327(94,8 \%)$ \\
\hline $16-50$ años ... & $112(32,5 \%)$ & BRAZO UTILIZADO & \\
\hline 51-65 años . & $233(67,5 \%)$ & Único ..... & $51(14,8 \%)$ \\
\hline NIVEL DE ESTUDIOS & & Ambos............................... & $294(85,2 \%)$ \\
\hline Sin Estudios/Primarios ........................... & $187(54,2 \%)$ & BRAZO DOMINANTE & \\
\hline Secundarios/FP/ Superiores.............. & $158(45,8 \%)$ & Derecho .......................... & $333(96,5 \%)$ \\
\hline DISTRIBUCIÓN POR ACTIVIDAD & & Izquierdo .. & $12(3,5 \%)$ \\
\hline ECONÓMICA & & ALZAR HOMBRO & \\
\hline Construcción, Agricultura, & & 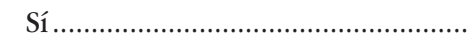 & $259(75,1 \%)$ \\
\hline Ganadería y Pesca ................ & $24(7,0 \%)$ & HERRAMIENTAS VIBRATORIAS & \\
\hline Comercio y Hostelería......... & $42(12,2 \%)$ & Sí.. & $60(17,4 \%)$ \\
\hline Sanidad y Servicios sociales.. & $29(8,4 \%)$ & GOLPEAR SUPERFICIES & \\
\hline Transporte y almacén.............. & $29(8,4 \%)$ & 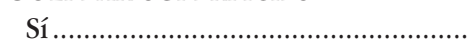 & $44(12,8 \%)$ \\
\hline Limpieza .................. & $122(35,4 \%)$ & POSTURA DE TRABAJO & \\
\hline Servicios........ & $30(8,7 \%)$ & Sentado & $60(17,4 \%)$ \\
\hline Industria ... & $26(7,5 \%)$ & 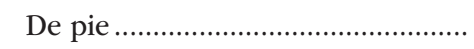 & $132(38,3 \%)$ \\
\hline Administración, banca y seguros. & & Alternados y cuclillas ........... & $153(44,3 \%)$ \\
\hline Enseñanza...... & $43(12,5 \%)$ & GIRAR CUELLO-COLUMNA & \\
\hline DISTRIBUCIÓN POR OCUPACIÓN & & 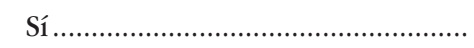 & $318(92,2 \%)$ \\
\hline 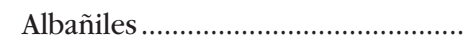 & $19(5,5 \%)$ & CARGAS > $15 \mathrm{KG}$ & \\
\hline 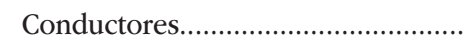 & $17(4,9 \%)$ & Sí.. & $105(30,4 \%)$ \\
\hline 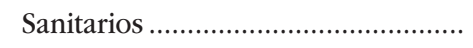 & $25(7,2 \%)$ & USO FUERZA FÍSICA & \\
\hline 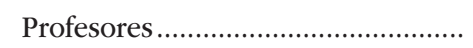 & $10(2,9 \%)$ & Sí.. & $57(16,5 \%)$ \\
\hline Limpiadoras ........ & $134(38,8 \%)$ & MOVIMIENTOS REPETITIVOS & \\
\hline Comerciales . & $35(10,1 \%)$ & Sí... & $315(91,3 \%)$ \\
\hline Administrativos y directivos.............. & $45(13,0 \%)$ & TRABAJO MONÓTONO & \\
\hline Artesanos, agricultores y ganaderos. & $23(6,7 \%)$ & 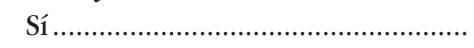 & $153(44,3 \%)$ \\
\hline Defensa y seguridad............................. & $5(1,4 \%)$ & PAUSAS & \\
\hline Mecánicos, soldadores, montadores & & Sí... & $303(87,8 \%)$ \\
\hline  & $23(6,7 \%)$ & DURACIÓN DE LAS PAUSAS & \\
\hline Informáticos e ingenieros .................... & $9(2,6 \%)$ & $<30$ minutos.............................. & $162(53,5 \%)$ \\
\hline HORAS DE TRABAJOS DIARIAS & & 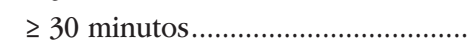 & $141(46,5 \%)$ \\
\hline$\geq 9 \mathrm{~h}$ & $28(8,1 \%)$ & POSIBLES AUSENCIAS & \\
\hline $8 \mathrm{~h}$ & $170(49,3 \%)$ & 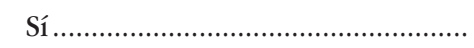 & $236(68,4 \%)$ \\
\hline$\leq 7 \mathrm{~h}$ & $147(42,6 \%)$ & LATERALIDAD & \\
\hline HORAS DE TRABAJO SEMANALES & & Derecho ........... & $201(58,3 \%)$ \\
\hline$>40 \mathrm{~h}$ & $32(9,3 \%)$ & Izquierdo ......................... & $106(30,7 \%)$ \\
\hline $40 \mathrm{~h} \ldots$ & $170(49,3 \%)$ & Ambos... & $38(11,0 \%)$ \\
\hline$<40 \mathrm{~h} \ldots$ & $143(41,4 \%)$ & INCAPACIDAD TEMPORAL (IT) & \\
\hline INFORMADO RIESGOS & & 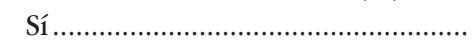 & $135(39.1 \%)$ \\
\hline Sí & $168(48,7 \%)$ & BAJA LABORAL/CONTROL & \\
\hline EQUIPOS DE PROTECCIÓN & & 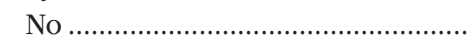 & $210(60.9 \%)$ \\
\hline  & $149(43,2 \%)$ & 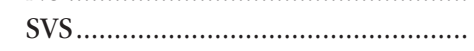 & $94(27.2 \%)$ \\
\hline SERVICIO DE PREVENCIÓN & & Mutua Laboral........................ & $41(11.9 \%)$ \\
\hline 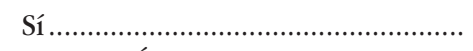 & $169(49,0 \%)$ & TIPO DE CONTINGENCIA & \\
\hline EVALUACIÓN DE RIESGOS & & Accidente de trabajo.......... & $40(11.6 \%)$ \\
\hline Sí $\ldots . . .$. & $135(39,1 \%)$ & Enfermedad profesional... & $4(1.2 \%)$ \\
\hline PLAN DE PREVENCIÓN & & Enfermedad común ........ & $300(87.0 \%)$ \\
\hline Sí .................. & $134(38,8 \%)$ & Accidente no laboral. & $1(0.3 \%)$ \\
\hline
\end{tabular}




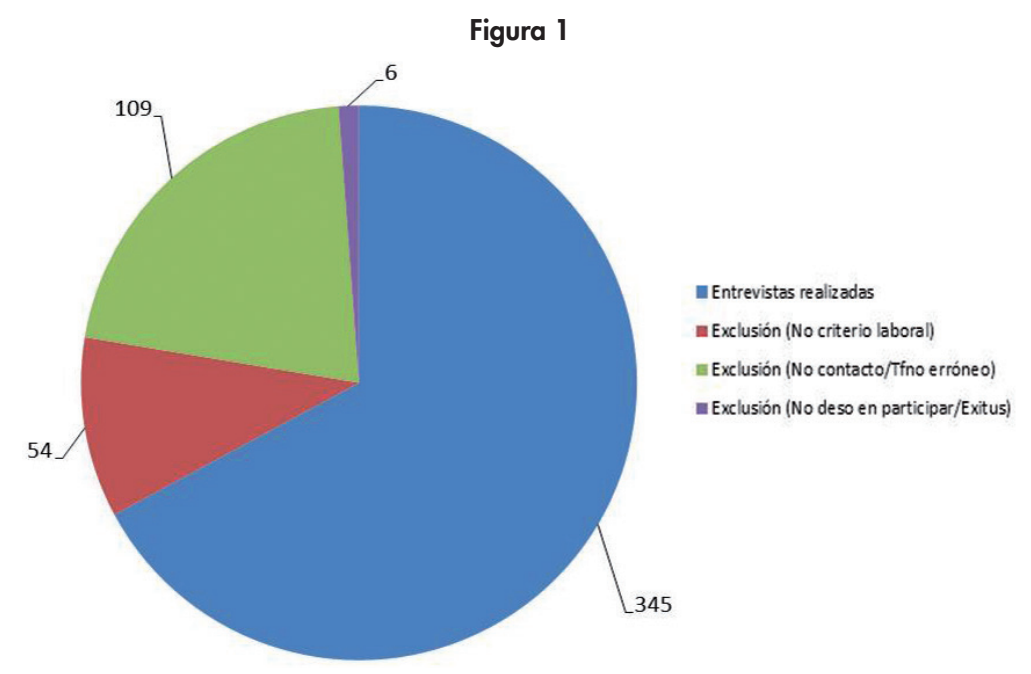

\section{RESULTADOS}

El $67 \%$ de los participantes eran mujeres, en un 54\% de los casos con estudios primarios o sin estudios, y dos terceras partes con edades comprendidas entre los 51 y los 65 años. La ocupación más numerosa correspondió a las limpiadoras seguida por personal administrativo o directivo, con jornadas laborales de $8 \mathrm{~h} /$ diarias y $40 \mathrm{~h} /$ semanales en casi la mitad de las ocasiones. El 39,1\% de los entrevistados presentaron un periodo de IT derivada, siendo en el $87 \%$ de los casos calificada como enfermedad común, y controlada en su mayoría por la Seguridad Social. En cuanto al nivel de conocimientos sobre prevención de riesgos laborales, el $51 \%$ de los encuestados refirió no haber recibido información sobre los riesgos en sus puestos ni disponer de un servicio de prevención asociado a la empresa, en torno al 60\% desconocía la existencia de evaluaciones de riesgos o planes de prevención; no obstante, el $53 \%$ afirmaron realizarse reconocimientos médicos periódicos. Los requerimientos ergonómicos más frecuentemente referidos en los puestos de trabajo eran (en $>75 \%$ de las ocasiones): realizar posturas forzadas, alzar el brazo por encima del nivel del hombro, girar el cuello o la columna, y la realización de movimientos repetidos (Tabla I).

Se asoció a una mayor probabilidad de presentar una baja laboral por patología del hombro: las actividades relacionadas con la construcción, la agricultura, ganadería y pesca, el conocimiento de la existencia de evaluación de riesgos y/o plan de prevención en la empresa, así como requerir en el puesto de trabajo alzar los brazos por encima del hombro, golpear superficies, manejar cargas $>15 \mathrm{~kg}$, y el uso de fuerza física en el trabajo (Tablas II-IV).

Tras el análisis multivariante, el conocimiento de la existencia de un plan de prevención en la empresa se mostró como un factor de riesgo independiente para presentar una baja laboral por patología no traumática del hombro (OR 4,2 [1,1-15,9]) (Tabla III).

\section{DISCUSIÓN}

Las bajas laborales asociadas a patología del hombro dependen de muchos factores que interactúan unos con otros. Según nuestros resultados, se asociaron a una mayor probabilidad de presentar una IT las actividades relacionadas con la construcción, la agricultura, ganadería y pesca, requerimientos ergonómicos exigentes como alzar los brazos por encima del nivel del hombro, golpear superficies, manejar cargas superiores a $15 \mathrm{~kg}$, y el uso de fuerza física en el trabajo, así como referir conocimientos preventivos sobre la evaluación de riesgos y el plan de prevención en la empresa. Los trastornos del hombro pueden suponer pérdidas importantes de productividad laboral, sin embargo, la información disponible sobre los factores determinantes y predictores de las bajas laborales es escasa e inconsistente ${ }^{17,18}$. 
Tabla II. Análisis de la asociación entre variables sociodemográficas y bajas laborales en los casos estudiados

\begin{tabular}{|c|c|c|c|c|c|c|}
\hline & $\begin{array}{l}\text { Con Baja } \\
(n=135)\end{array}$ & $\begin{array}{l}\text { Sin Baja } \\
(\mathrm{n}=210)\end{array}$ & OR (IC 95\%) & $\mathbf{P}$ & ORa (IC 95\%) & $\mathbf{P}$ \\
\hline \multicolumn{7}{|l|}{ Sexo } \\
\hline  & $34,1 \%(46)$ & $31,0 \%(65)$ & $1,1(0,7-1,8)$ & N.S. & - & - \\
\hline 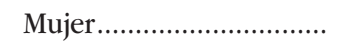 & $65,9 \%(89)$ & $69,0 \%(145)$ & & & & \\
\hline \multicolumn{7}{|l|}{ Edad } \\
\hline $51-65 \ldots \ldots \ldots \ldots \ldots \ldots \ldots \ldots$ & $63,7 \%(86)$ & $70,0 \%(147)$ & $0,7(0,5-1,2)$ & N.S. & - & - \\
\hline $16-50$ & $36,3 \%(49)$ & $30,0 \%(63)$ & 1 & & & \\
\hline \multicolumn{7}{|l|}{ Nivel de estudios } \\
\hline $\begin{array}{l}\text { Sin estudios/Primarios .. } \\
\text { Secundarios/Formación }\end{array}$ & $54,8 \%(74)$ & $53,8 \%(113)$ & $1,0(0,7-1,6)$ & N.S. & - & - \\
\hline Profesional/Superiores.. & $45,2 \%(61)$ & $46,2 \%(97)$ & 1 & & & \\
\hline \multicolumn{7}{|l|}{$\begin{array}{l}\text { Construcción, } \\
\text { Agricultura, Ganadería y }\end{array}$} \\
\hline Pesca .......................................... & $12,6 \%(17)$ & $3,3 \%(7)$ & $7,1(2,3-21,5)$ & 0,001 & $3,3(0,8-13,9)$ & 0,097 \\
\hline $\begin{array}{l}\text { Comercio y Hostelería... } \\
\text { Sanidad y Servicios }\end{array}$ & $11,9 \%(16)$ & $12,4 \%(26)$ & $1,8(0,7-4,5)$ & N.S. & $1,7(0,6-4,9)$ & N.S. \\
\hline 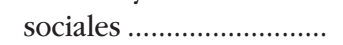 & $7,4 \%(10)$ & $9,0 \%(19)$ & $1,5(0,5-4,3)$ & N.S. & $1,6(0,5-5,3)$ & N.S. \\
\hline Transporte y almacén.... & $8,1 \%(11)$ & $8,6 \%(18)$ & $1,8(0,6-4,9)$ & N.S. & $1,5(0,5-4,6)$ & N.S. \\
\hline 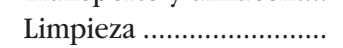 & $35,6 \%(48)$ & $35,2 \%(74)$ & $1,9(0,9-4,1)$ & 0,108 & $2,1(0,8-5,5)$ & 0,123 \\
\hline  & $8,9 \%(12)$ & $8,6 \%(18)$ & $1,9(0,7-5,3)$ & 0,195 & $1,4(0,5-4,3)$ & N.S. \\
\hline 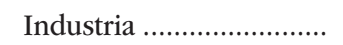 & $7,4 \%(10)$ & $7,6 \%(16)$ & $1,8(0,6-5,2)$ & N.S. & $1,7(0,5-5,8)$ & N.S. \\
\hline $\begin{array}{l}\text { Administración, banca y } \\
\text { seguros. Enseñanza ........ }\end{array}$ & $8,1 \%$ (11) & $15,2 \%(32)$ & 1 & & & \\
\hline \multicolumn{7}{|c|}{ Ocupación } \\
\hline Albañiles ... & $9,6 \%(13)$ & $2,9 \%(6)$ & $4,3(0,8-23,5)$ & 0,089 & - & - \\
\hline Conductores....................... & $5,9 \%(8)$ & $4,3 \%(9)$ & $1,8(0,3-9,5)$ & N.S. & - & - \\
\hline 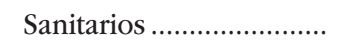 & $5,2 \%(7)$ & $8,6 \%(18)$ & $0,8(0,1-4,0)$ & N.S. & - & - \\
\hline Profesores............................ & $0 \%(0)$ & $4,8 \%(10)$ & $0,0(0,0-114,49)$ & N.S. & - & - \\
\hline Limpiadoras .......................... & $39,3 \%(53)$ & $38,6 \%(81)$ & $1,3(0,3-5,5)$ & N.S. & - & - \\
\hline Comerciales ........................ & $9,6 \%(13)$ & $10,5 \%(22)$ & $1,2(0,2-5,5)$ & N.S. & - & - \\
\hline 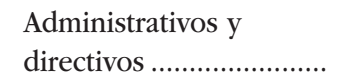 & $11,1 \%(15)$ & $14,3 \%(30)$ & $1,0(0,2-4,6)$ & N.S. & - & - \\
\hline $\begin{array}{l}\text { Artesanos, agricultores y } \\
\text { ganaderos }\end{array}$ & $6,7 \%(9)$ & $6,7 \%(14)$ & $1,3(0,2-6,5)$ & N.S. & - & - \\
\hline Defensa y seguridad....... & $0,7 \%(1)$ & $1,9 \%(4)$ & $0,5(0,0-8,7)$ & N.S. & - & - \\
\hline $\begin{array}{l}\text { Mecánicos, soldadores, } \\
\text { montadores industriales }\end{array}$ & $9,6 \%(13)$ & $4,8 \%(10)$ & $2,6(0,5-13,0)$ & N.S. & - & - \\
\hline $\begin{array}{l}\text { Informáticos e } \\
\text { ingenieros ........................... }\end{array}$ & $2,2 \%(3)$ & $2,9 \%(6)$ & 1 & & & \\
\hline \multicolumn{7}{|l|}{ Horas trabajo diarias } \\
\hline$\geq 9 \mathrm{~h}$ & $8,9 \%(12)$ & $7,6 \%(16)$ & $1,2(0,5-2,8)$ & N.S. & - & - \\
\hline 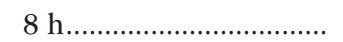 & $49,6 \%(67)$ & $49,0 \%(103)$ & $1,1(0,7-1,7)$ & N.S. & - & - \\
\hline$\leq 7 \mathrm{~h}$ & $41,5 \%(56)$ & $43,3 \%(91)$ & 1 & & & \\
\hline \multicolumn{7}{|l|}{ Horas trabajo semanales } \\
\hline$>40 \mathrm{~h}$ & $11,9 \%(16)$ & $7,6 \%(16)$ & $1,6(0,7-3,5)$ & N.S. & - & - \\
\hline $40 \mathrm{~h} \ldots \ldots$ & $47,6 \%(64)$ & $50,5 \%(106)$ & $1,0(0,6-1,5)$ & N.S. & - & - \\
\hline$<40$ h.... & $40,7 \%(55)$ & $41,9 \%(88)$ & 1 & & & \\
\hline
\end{tabular}

N.S.: $p>0.200$. Ajustado por: Actividad económica, Información de los riesgos, Equipos de protección,

Servicio de prevención, Evaluación de riesgos, Plan de prevención, Brazo utilizado, Alzar el hombro, Herramientas vibratorias, Golpear superficies, Cargas $>15 \mathrm{Kg}$, Uso de fuerza física.

Los requerimientos ergonómicos que aparecen asociados a las bajas laborales por patología no traumática del hombro podrían actuar de manera aguda provocando sobreesfuerzos de la articulación, y así requerir de períodos de incapacidad laboral temporal para su recuperación. Nuestros hallazgos coinciden con otros estudios que identifican el manejo de cargas pesadas, alzar los brazos por encima del hombro, y la sobrecarga física percibida en el trabajo como factores predictores de bajas laborales de 
larga duración por trastornos musculoesqueléticos ${ }^{19,20}$. De hecho, golpear superficies, alzar los brazos por encima del hombro y el brazo utilizado presentan en nuestro estudio una clara tendencia a la significación de manera independiente; sin embargo, no encontramos diferencias estadísticamente significativas en cuanto al sexo y la edad de los participantes, a diferencia de otros autores ${ }^{20}$. Otros factores que se han descrito en la literatura asociados a ausencias laborales o retraso en la reincorporación laboral por patología del hombro son: el antecedente no traumático, gravedad de la lesión, bajas laborales previas, intensidad del dolor, factores psicológicos y el hábito tabáquico ${ }^{17,18,21}$. No obstante, las discrepancias en los resultados deben interpretarse con cautela por posibles limitaciones en la comparabilidad entre estudios debido a diferencias en cuanto a diseños, tamaños de muestra o recogida de variables y su aplicación en contextos culturales distintos.

Tabla III. Análisis de la asociación entre conocimientos sobre prevención de los riesgos del puesto de trabajo y presencia de bajas laborales en los casos estudiados

\begin{tabular}{|c|c|c|c|c|c|c|}
\hline & $\begin{array}{l}\text { Con Baja } \\
(\mathrm{n}=135)\end{array}$ & $\begin{array}{l}\text { Sin Baja } \\
(\mathrm{n}=\mathbf{2 1 0})\end{array}$ & OR (IC 95\%) & $\mathbf{P}$ & ORa (IC 95\%) & $\mathbf{P}$ \\
\hline \multicolumn{7}{|l|}{ Información riesgos } \\
\hline Sí..... & $55,6 \%(75)$ & $44,3 \%(93)$ & $1,6(1,0-2,4)$ & 0,041 & $1,1(0,5-2,3)$ & N.S. \\
\hline No ...................................... & $44,4 \%(60)$ & $55,7 \%(117)$ & 1 & & 1 & \\
\hline \multicolumn{7}{|l|}{ Equipos de protección } \\
\hline 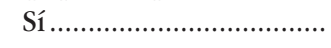 & $50,4 \%(68)$ & $38,6 \%(81)$ & $1,6(1,0-2,5)$ & 0,031 & $0,9(0,5-1,8)$ & N.S. \\
\hline 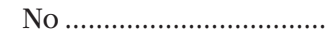 & $49,6 \%(67)$ & $61,4 \%(129)$ & 1 & & 1 & \\
\hline \multicolumn{7}{|l|}{ Servicio de Prevención } \\
\hline 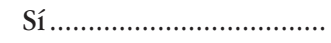 & $56,3 \%(76)$ & $44,3 \%(93)$ & $1,6(1,0-2,5)$ & 0,029 & $1,1(0,4-3,0)$ & N.S. \\
\hline No …............................ & $43,7 \%(59)$ & $55,7 \%(117)$ & 1 & & 1 & \\
\hline \multicolumn{7}{|l|}{ Evaluación de riesgos } \\
\hline 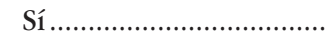 & $46,7 \%(63)$ & $34,3 \%(72)$ & $1,7(1,1-2,6)$ & 0,021 & $0,5(0,1-1,7)$ & N.S. \\
\hline  & $53,3 \%(72)$ & $65,7 \%(138)$ & 1 & & 1 & \\
\hline \multicolumn{7}{|l|}{ Plan de prevención } \\
\hline 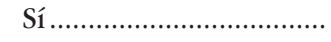 & $48,9 \%(66)$ & $32,4 \%(68)$ & $2,0(1,3-3,1)$ & 0,002 & $4,2(1,1-15,9)$ & 0,036 \\
\hline No ....... & $51,1 \%(69)$ & $67,6 \%(142)$ & 1 & & 1 & \\
\hline \multicolumn{7}{|l|}{ Vigilancia de salud } \\
\hline 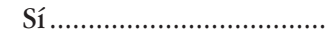 & $57,8 \%(78)$ & $50,5 \%(106)$ & $1,3(0,9-2,1)$ & 0,185 & - & - \\
\hline 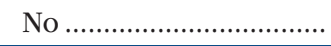 & $42,2 \%(57)$ & $49,5 \%(104)$ & 1 & & & \\
\hline
\end{tabular}

N.S.: $p>$ 0.200. Ajustado por: Actividad económica, Información de los riesgos, Equipos de protección

Servicio de prevención, Evaluación de riesgos, Plan de prevención, Brazo utilizado, Alzar el hombro, Herramientas vibratorias, Golpear superficies, Cargas $>15 \mathrm{Kg}$, Uso de fuerza física.

De acuerdo a nuestros resultados, los sectores de actividad laboral que presentaron mayor riesgo de sufrir IT por motivo del hombro realizan tareas con demandas físicas exigentes. Así, el sector de la construcción se caracteriza por exposición a: manipulación de cargas pesadas y utilización de fuerza física ${ }^{19}$. En un estudio realizado en un colectivo de vendimiadores (dentro del sector agrícola) se han identificado factores biomecánicos y psicosociales de las tareas asociados a trastornos musculoesqueléticos de miembro superior, como: insuficientes medios materiales, bajo nivel de control y un desequilibrio entre esfuerzos y recompensas ${ }^{22}$.

En cuanto a la información que refiere conocer el trabajador acerca de los recursos preventivos de la empresa y riesgos del puesto de trabajo, los resultados observados no concuerdan con lo que cabría esperar. Parecería que actuaciones integradas en prevención como son la identificación y evaluación de los riesgos del puesto, así como disponer de una planificación de las actividades preventivas en la empresa, no actuarían como factores de riesgo para precisar una baja laboral. De hecho, tras el análisis multivariante, la única variable que mostró un efecto independiente fue disponer de un plan de prevención. Este resultado a priori resulta paradójico, porque esperaríamos lo contrario. A este respecto, no se ha encontrado evidencia en la literatura que apoye o refute dichos resultados, aunque podría tener relación con un mayor conocimiento de las estructuras preventivas 
y protectoras frente a la enfermedad y una mayor sensibilización frente a la misma. Más bien, las orientaciones se dirigen hacia un abordaje preventivo laboral amplio. Existe evidencia de calidad moderada en la realización de intervenciones en el puesto de trabajo para reducir el número de ausencias por enfermedad entre trabajadores con trastornos musculoesqueléticos ${ }^{23}$. Además, al tener la patología de hombro un origen multifactorial, las intervenciones preventivas que incluyan los factores físicos, psicosociales y organizativos probablemente sean las que presenten mayores efectos ${ }^{13,24,25}$. También existe un amplio consenso en buscar una vuelta temprana al trabajo, aunque en muchas ocasiones sea necesario realizar algún tipo de adaptación del puesto ${ }^{13}$.

Tabla IV. Análisis de la asociación entre requerimientos ergonómicos del puesto de trabajo y presencia de bajas laborales

\begin{tabular}{|c|c|c|c|c|c|c|}
\hline & $\begin{array}{l}\text { Con Baja } \\
(\mathrm{n}=135)\end{array}$ & $\begin{array}{l}\text { Sin Baja } \\
(\mathrm{n}=\mathbf{2 1 0})\end{array}$ & OR (IC 95\%) & $\mathbf{P}$ & ORa (IC 95\%) & $\mathbf{P}$ \\
\hline \multicolumn{7}{|l|}{ Posturas forzadas } \\
\hline Sí ........................ & $94,8 \%(128)$ & $94,8 \%(199)$ & $1,0(0,4-2,7)$ & N.S. & - & - \\
\hline No ....... & $5,2 \%(7)$ & $5,2 \%$ (11) & 1 & & & \\
\hline \multicolumn{7}{|l|}{ Brazo utilizado } \\
\hline Único ................. & $10,4 \%(14)$ & $17,6 \%(37)$ & $0,5(0,3-1,0)$ & 0,064 & $0,5(0,3-1,1)$ & 0,106 \\
\hline Ambos................... & $89,6 \%(121)$ & $82,4 \%(173)$ & 1 & & 1 & \\
\hline \multicolumn{7}{|l|}{ Brazo dominante } \\
\hline Derecho ............... & $97,0 \%(131)$ & $96,2 \%(202)$ & $1,3(0,4-4,4)$ & N.S. & - & - \\
\hline Izquierdo .............................. & $3,0 \%(4)$ & $3,8 \%(8)$ & 1 & & & \\
\hline \multicolumn{7}{|l|}{ Alzar hombro } \\
\hline Sí .................... & $84,4 \%(114)$ & $69,0 \%(145)$ & $2,4(1,4-4,2)$ & 0,001 & $1,8(0,9-3,6)$ & 0,089 \\
\hline  & $15,6 \%(21)$ & $31,0 \%(65)$ & 1 & & 1 & \\
\hline \multicolumn{7}{|c|}{\begin{tabular}{l|l} 
Herramientas vibratorias & 1,070 (1)
\end{tabular}} \\
\hline Sí & $22,2 \%(30)$ & $14,3 \%(30)$ & $1,7(1,0-3,0)$ & 0,058 & $0,7(0,3-1,8)$ & N.S. \\
\hline 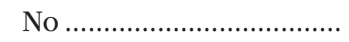 & $77,8 \%(105)$ & $85,7 \%(180)$ & 1 & & 1 & \\
\hline \multicolumn{7}{|l|}{ Golpear superficies } \\
\hline Sí & $20,7 \%(28)$ & $7,6 \%(16)$ & $3,2(1,6-6,1)$ & $<0,001$ & $2,4(0,8-7,4)$ & 0,111 \\
\hline No .......................... & $79,3 \%(107)$ & $92,4 \%(194)$ & 1 & & 1 & \\
\hline \multicolumn{7}{|l|}{ Postura de trabajo } \\
\hline Sentado........ & $13,3 \%(18)$ & $20,0 \%(42)$ & $0,6(0,3-1,2)$ & 0,133 & - & - \\
\hline De pie.... & $40,0 \%(54)$ & $37,1 \%(78)$ & $1,0(0,6-1,6)$ & N.S. & & \\
\hline Alternados y cuclillas ....... & $46,7 \%(63)$ & $42,9 \%(90)$ & 1 & & & \\
\hline \multicolumn{7}{|l|}{ Girar cuello-columna } \\
\hline Sí .......... & $94,1 \%(127)$ & $91,0 \%(191)$ & $1,6(0,7-3,7)$ & N.S. & - & - \\
\hline No ........... & $5,9 \%(8)$ & $9,0 \%(19)$ & 1 & & & \\
\hline \multicolumn{7}{|l|}{ Cargas > $15 \mathrm{Kg}$} \\
\hline Sí $\ldots \ldots \ldots . . . .$. & $38,5 \%(52)$ & $25,2 \%(53)$ & $1,9(1,2-3,0)$ & 0,009 & $1,1(0,6-2,1)$ & N.S. \\
\hline No ........................... & $61,5 \%(83)$ & $74,8 \%(157)$ & 1 & & & \\
\hline \multicolumn{7}{|l|}{ Uso fuerza físíca } \\
\hline 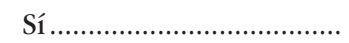 & $24,4 \%(33)$ & $11,4 \%(24)$ & $2,5(1,4-4,5)$ & 0,001 & $1,1(0,5-2,7)$ & N.S. \\
\hline No ................................................. & $75,6 \%(102)$ & $88,6 \%(186)$ & 1 & & & \\
\hline \multicolumn{7}{|l|}{ Movimientos repetitivos } \\
\hline 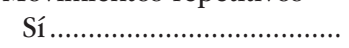 & $93,3 \%(126)$ & $90,0 \%(189)$ & $1,6(0,7-3,5)$ & N.S. & - & - \\
\hline No & $6,7 \%(9)$ & $10,0 \%(21)$ & 1 & & & \\
\hline \multicolumn{7}{|l|}{ Trabajo monótono } \\
\hline 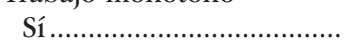 & $48,1 \%(65)$ & $41,9 \%(88)$ & $1,3(0,8-2,0)$ & N.S. & - & - \\
\hline No .......................................... & $51,9 \%(70)$ & $58,1 \%(122)$ & 1 & & & \\
\hline \multicolumn{7}{|l|}{ Pausas } \\
\hline 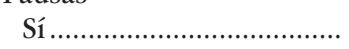 & $98,6 \%(121)$ & $86,7 \%(182)$ & $1,3(0,7-2,6)$ & N.S. & - & - \\
\hline No & $10,4 \%(14)$ & $13,3 \%(28)$ & & & & \\
\hline \multicolumn{7}{|l|}{ Duración de las pausas } \\
\hline$<30$ minutos......................... & $50,4 \%(61)$ & $55,5 \%(101)$ & $0,8(0,5-1,3)$ & N.S. & - & - \\
\hline$\geq 30$ minutos ............ & $49,6 \%(60)$ & $44,5 \%(81)$ & & & & \\
\hline \multicolumn{7}{|l|}{ Posibles ausencias } \\
\hline Sí & $71,9 \%(97)$ & $66,2 \%(139)$ & $1,3(0,8-2,1)$ & N.S. & - & - \\
\hline 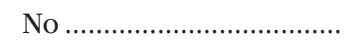 & $28,1 \%(38)$ & $33,8 \%(71)$ & 1 & & & \\
\hline
\end{tabular}

N.S.: $p$ > 0.200. Ajustado por: Actividad económica, Información de los riesgos, Equipos de protección Servicio de prevención, Evaluación de riesgos, Plan de prevención, Brazo utilizado, Alzar el hombro, Herramientas vibratorias, Golpear superficies, Cargas $>15 \mathrm{Kg}$, Uso de fuerza física. 
Respecto a las limitaciones de este estudio, son las propias de un diseño transversal. La información procede de un centro de salud del Departamento de salud de Alicante, con una tasa media de respuestas del $67,1 \%$, por lo que no podemos asegurar la representatividad de la muestra y hay que ser prudentes a la hora de extrapolar los resultados. También la recogida de información sobre la exposición retrospectivamente a la presencia de enfermedad puede suponer un posible sesgo de memoria en los participantes y ambigüedad temporal. Los requerimientos ergonómicos (así como el resto de información recogida en la encuesta) se basan en la percepción referida por los trabajadores en función de las descripciones de tareas realizadas en sus puestos, y no proceden de los requerimientos reales observados en las evaluaciones de riesgos de dichos puestos, por lo que los resultados podrían perder relevancia. Otras limitaciones son: una codificación diagnóstica errónea en casos atendidos que no hayan sido incluidos y limitaciones inherentes a la encuesta, al no haberse documentado antecedentes como: lesiones deportivas/aficiones que impliquen el uso de los hombros, o las tareas domésticas, deportivas o de ocio, que podrían relacionarse con el origen del dolor de hombro independientemente de una asociación laboral.

Más de un tercio de los participantes en este estudio presentaron un periodo de IT a causa de la patología no traumática del hombro. Dada la frecuencia del hombro doloroso, los costes derivados de su tratamiento, y el posible desarrollo de bajas laborales, es importante el abordaje preventivo. Tanto prevenir los requerimientos ergonómicos del puesto de trabajo que se asocian al desarrollo de IT (alzar los brazos por encima del nivel del hombro, golpear superficies, manejar cargas $>15 \mathrm{~kg}$, y el uso de fuerza física) como un mayor conocimiento del efecto de las estructuras preventivas en el proceso. Se necesitarían más estudios de mayor tamaño, ampliando el ámbito territorial y que incluyan las actividades extralaborales y los requerimientos ergonómicos identificados en las evaluaciones de riesgo de los puestos, para confirmar nuestros hallazgos.

\section{REFERENCIAS BIBLIOGRÁFICAS}

1. European Commission. Second stage of consultation of the social partners on work-related musculoskeletal disorders. Brussels: DG Employment, Social Affairs \& Inclusion; 2007.

2. Schneider E, Irastorza X, Copsey S. OSH [Occupational safety and health] in figures: work-related musculoskeletal disorders in the EU-facts and figures. Luxembourg: Publications Office of the European Union; 2010.

3. Instituto Nacional de Seguridad e Higiene en el Trabajo. VII Encuesta Nacional de Condiciones de Trabajo. Madrid: Ministerio de Empleo y Seguridad Social; 2011.

4. Lázaro P, Parody E, Méndez JI, Alfaro N, Gabriele J, García de Vicuña T, Jover JA, et al. También está en tu mano. Impacto de las enfermedades reumáticas en España. Madrid: Fundación Abbott; 2009.

5. Hämmig O, Knecht M, Läubli T, Bauer GF. Work-life conflict and musculoskeletal disorders: a crosssectional study of an unexplored association. BMC Musculoskelet Disord. 2011;12:60. https://doi. org/10.1186/1471-2474-12-60.

6. Dorrestijn O, Greving K, van der Veen WJ, van der Meer K, Diercks RL, Winters JC, et al. Patients with shoulder complaints in general practice: consumption of medical care. Rheumatology (Oxford). 2011;50(2):389-95. https://doi.org/10.1093/rheumatology/keq333.

7. Kuijpers T, van Tulder MW, van der Heijden GJ, Bouter LM, van der Windt DA. Costs of shoulder pain in primary care consulters: a prospective cohort study in The Netherlands. BMC Musculoskelet Disord. 2006;7:83. https://doi.org/10.1186/1471-2474-7-83.

8. Virta L, Joranger P, Brox JI, Eriksson R. Costs of shoulder pain and resource use in primary health care: a cost-of-illness study in Sweden. BMC Musculoskelet Disord. 2012;13:17. https://doi.org/10.1186/14712474-13-17.

9. Marin-Gomez M, Navarro-Collado MJ, Peiro S, Trenor-Gomis C, Paya-Rubio A, Bernal-Delgado E, et al. La calidad de la atención al hombro doloroso. Audit clínico. Gac Sanit. 2006;20(2):116-23.

10. Weevers HJ, van der Beek AJ, Anema JR, van der Wal G, van Mechelen W. Work-related disease in general practice: a systematic review. Fam Pract. 2005;22(2):197-204. https://doi.org/10.1093/fampra/cmh727 
11. Luime JJ, Koes BW, Hendriksen IJ, Burdorf A, Verhagen AP, Miedema HS, et al. Prevalence and incidence of shoulder pain in the general population; a systematic review. Scand J Rheumatol. 2004;33(2):73-81. https://doi.org/10.1080/03009740310004667

12. Murphy RJ, Carr AJ. Shoulder pain. BMJ Clin Evid. 2010 Jul 22. pii: 1107.

13. Shanahan EM, Sladek R. Shoulder pain at the workplace. Best Pract Res Clin Rheumatol. 2011;25(1):59-68. https://doi.org/10.1016/j.berh.2011.01.008.

14. Herin F, Vézina M, Thaon I, Soulat J-M, Paris C. Predictors of chronic shoulder pain after 5 years in a working population. Pain. 2012;153(11):2253-9. https://doi.org/10.1016/j.pain.2012.07.024

15. Svendsen SW, Bonde JP, Mathiassen SE, Stengaard-Pedersen K, Frich L. Work related shoulder disorders: quantitative exposure-response relations with reference to arm posture. Occup Environ Med. 2004;61(10):844-53. https://doi.org/10.1136/oem.2003.010637.

16. Organización Mundial de la Salud. Identificación de enfermedades relacionadas con el trabajo y medidas para combatirlas: informe de un Comité de Expertos de la OMS. Ginebra: Organización Mundial de la Salud; 1985.

17. Desmeules F, Braën C, Lamontagne M, Dionne CE, Roy JS. Determinants and predictors of absenteeism and return-to-work in workers with shoulder disorders. Work. 2016;55(1):101-13. https://doi.org/10.3233/ WOR-162379

18. Kuijpers T, van der Windt DA, van der Heijden GJ, Twisk JW, Vergouwe Y, Bouter LM. A prediction rule for shoulder pain related sick leave: a prospective cohort study. BMC Musculoskelet Disord. 2006;7:97. https://doi.org/10.1186/1471-2474-7-97

19. Holtermann A, Jørgensen MB, Gram B, Christensen JR, Faber A, Overgaard K, et al. Worksite interventions for preventing physical deterioration among employees in job-groups with high physical work demands: background, design and conceptual model of FINALE. BMC Public Health. 2010;10:120. https://doi. org/10.1186/1471-2458-10-120.

20. Lötters F, Burdorf A. Prognostic factors for duration of sickness absence due to musculoskeletal disorders. Clin J Pain. 2006;22(2):212-21. https://doi.org/10.1097/01.ajp.0000154047.30155.72

21. Holtermann A, Hansen JV, Burr H, Søgaard K. Prognostic factors for long-term sickness absence among employees with neck-shoulder and low-back pain. Scand J Work, Environ Health. 2010;36(1):34-41. https://doi.org/10.5271/sjweh.2883

22. Bernard C, Courouve L, Bouée S, Adjémian A, Chrétien J-C, Niedhammer I. Biomechanical and psychosocial work exposures and musculoskeletal symptoms among vineyard workers. J Occup Health. 2011;53(5):297-311. https://doi.org/10.1539/joh.10-0031-oa

23. van Oostrom SH, Driessen MT, de Vet HC, Franche RL, Schonstein E, Loisel P, et al. Workplace interventions for preventing work disability. Cochrane Database Syst Rev. 2009 Apr 15;(2):CD006955. https://doi.org/10.1002/14651858.CD006955.pub2.

24. Larsson B, Søgaard K, Rosendal L. Work related neck-shoulder pain: a review on magnitude, risk factors, biochemical characteristics, clinical picture and preventive interventions. Best Pract Res Clin Rheumatol. 2007;21(3):447-63. https://doi.org/10.1016/j.berh.2007.02.015

25. Halpern M, Hurd JL, Zuckerman JD. Occupational shoulder disorders. En: Rockwood Ch, Matsen FA, editors. The shoulder. 4th Edition. Philadelphia: Saunders Elsevier; 2009. p. 1489-1508.

|| 